\title{
The QWeak Experiment: A measurement of the proton weak charge and up-down quark weak couplings.
}

\author{
Michael Gericke*t \\ University of Manitoba \\ E-mail: moerickeephysics.umanitoba.ca
}

In May 2012, the $Q_{W e a k}^{p}$ collaboration completed a two year measurement program to determine the weak charge of the proton $Q_{W}^{p}=\left(1-4 \sin ^{2} \theta_{W}\right)$ at the Thomas Jefferson National Accelerator Facility (TJNAF). The experiment was designed to produce a $4.0 \%$ measurement of the weak charge, via a $2.5 \%$ measurement of the parity violating asymmetry in the number of elastically scattered $1.165 \mathrm{GeV}$ electrons from protons, at forward angles. At the proposed precision, the experiment would produce a $0.3 \%$ measurement of the weak mixing angle at a momentum transfer of $Q^{2}=0.026 \mathrm{GeV}^{2}$, making it the most precise stand alone measurement of the weak mixing angle at low momentum transfer. In combination with other parity measurements, $Q_{\text {Weak }}^{p}$ will also provide a high precision determination of the weak charges of the up and down quarks. At the proposed precision, a significant deviation from the Standard Model prediction could be a signal of new physics at mass scales up to $\simeq 6 \mathrm{TeV}$, whereas agreement would place new and significant constraints on possible Standard Model extensions at mass scales up to $\simeq 2 \mathrm{TeV}$. This paper provides an overview of the physics and the experiment, as well as a brief look at some preliminary diagnostic and analysis data. First results have recently been published for our commissioning run, which constitutes about $4 \%$ of our collected data [四].

The European Physical Society Conference on High Energy Physics

18-24 July, 2013

Stockholm, Sweden

\footnotetext{
* Speaker.

${ }^{\dagger}$ For the QWeak Collaboration
} 
The subject of this paper is the $Q_{W e a k}^{p}$ experiment, which completed a two year long measurement program in May 2012, in Hall C at the Thomas Jefferson National Accelerator Facility (TJNAF), to measure the weak charge of the proton $Q_{W}^{p}=1-4 \sin ^{2} \theta_{W} \simeq 0.075$. The weak charge of the proton is a suppressed quantity in the SM and results from this experiment, in conjunction with previous and future measurements of parity-violating electron scattering, will constrain the possibility of relevant physics beyond the Standard Model to the multi-TeV energy scale. The $Q_{\text {Weak }}^{p}$ experiment started data production in fall 2010. The $Q_{\text {Weak }}^{p}$ collaboration recently published the commissioning run results, constituting $4 \%$ of the total data volume [U] and the relevant physics

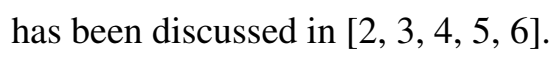

The experimental observable is the parity violating asymmetry in the number of elastically scattered electrons $\left(E_{\text {beam }}=1.165 \mathrm{GeV}\right)$ from protons at very forward angles $\left(\theta=8 \pm 2^{\circ}\right)$. The aim is a measurement of the asymmetry with a $\pm 2.1 \%$ statistical and $\pm 1.3 \%$ systematic uncertainty. In terms of the weak charges and nucleon form factors, the asymmetry is given by $[\text { [ }]^{1}$

$$
\begin{aligned}
A_{P V}(\vec{e}, p) & =k\left(Q^{2} Q_{W}^{p}+A_{H, V}+A_{H, A}\right) \\
A_{H, V} & =Q_{W}^{n} \frac{\varepsilon G_{E}^{p, \gamma} G_{E}^{n, \gamma}+\tau G_{M}^{p, \gamma} G_{M}^{n, \gamma}}{\varepsilon\left(G_{E}^{p, \gamma}\right)^{2}+\tau\left(G_{M}^{p, \gamma}\right)^{2}}+Q_{W}^{s} \frac{\varepsilon G_{E}^{p, \gamma} G_{E}^{s}+\tau G_{M}^{p, \gamma} G_{M}^{s}}{\varepsilon\left(G_{E}^{p, \gamma}\right)^{2}+\tau\left(G_{M}^{p, \gamma}\right)^{2}} \\
A_{H, A} & =Q_{W}^{e} \frac{\varepsilon^{\prime} G_{A}^{p, Z} G_{M}^{p, \gamma}}{\varepsilon\left(G_{E}^{p, \gamma}\right)^{2}+\tau\left(G_{M}^{p, \gamma}\right)^{2}} .
\end{aligned}
$$

At the chosen momentum transfer $\left(Q^{2}=0.026 \mathrm{GeV}^{2}\right)$, the SM predicted size of the asymmetry is $\simeq-230 \mathrm{ppb}$. Based on this prediction, the goal uncertainty is $\simeq \pm 6 \mathrm{ppb}$. Besides the proton weak charge, the asymmetry has contributions from hadronic form factors. At tree level, the first term in the asymmetry is proportional to the weak charge of the proton $Q_{W}^{p}=\left(1-4 \sin ^{2} \theta_{W}\right)$ and has a Standard Model predicted value of $\simeq-150 \mathrm{ppb}$. This term has contributions from radiative corrections, as well as from possible new physics. The hadronic portion of the asymmetry has two terms, corresponding to the vector and axial vector hadronic currents. These are parameterized in terms of the electromagnetic and strange form factors of the neutron and proton. At the chosen kinematics, the vector contribution to the asymmetry is about $\simeq-70 \mathrm{ppb}$, while the axial vector contribution is about $\simeq-10 \mathrm{ppb}$. The asymmetry decreases with momentum transfer, making the measurement at lower $Q^{2}$ harder. However, at this low $Q^{2}$, the weak charge contribution to the asymmetry has been maximized relative to the contributions from hadronic effects, while still allowing the measurement of the asymmetry within a reasonable time. The hadronic contributions are well constrained by the world data set [ [ 8, Q $⿴ 囗$ ].

The SM and extensive calculations of radiative corrections [1], 미, ㅁ], [2], [1], [4]] allow for a precise prediction of $Q_{W}^{p}$, as a function of the weak mixing angle, $\sin ^{2} \theta_{W}$, from the $Z^{0}$ pole down to low energies, as shown in Fig. $\amalg$. The precise measurements near the $Z^{0}$ pole anchor the curve at one particular energy scale. The shape of the curve away from this point is a prediction of the SM and to test this prediction one needs precise, off-peak measurements.

$$
{ }^{1} k \equiv-\frac{G_{F}}{4 \pi \alpha \sqrt{2}}, \varepsilon \equiv \frac{1}{1+2(1+\tau) \tan ^{2} \frac{\theta}{2}}, \varepsilon^{\prime} \equiv \sqrt{\tau(1+\tau)\left(1-\varepsilon^{2}\right)}, \text { and } \tau \equiv Q^{2} / 4 M_{N}^{2} .
$$


The QWeak Experiment: A measurement of the proton weak charge and up-down quark weak couplings. Michael Gericke
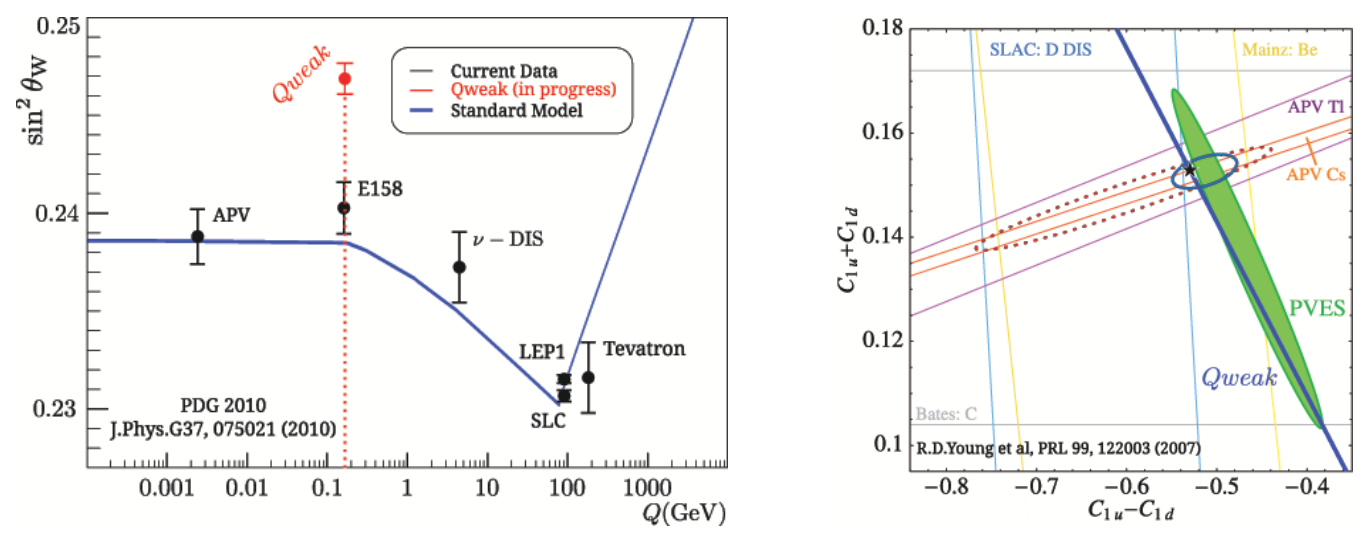

Figure 1: Left: Calculated running of the weak mixing angle in the Standard Model, as defined in the modified minimal subtraction scheme [[]]. The black error bars show completed measurements [15], [16, [17, [18], while the red error bar (with arbitrarily chosen vertical location) refers to the proposed $4 \% Q_{\text {Weak }}^{p}$ measurement. Right: Constraints on the neutral weak effective couplings to up and down quarks [Q] ]. The dotted contour displays the experimental limits (95\% CL) reported in the PDG [18] together with the prediction of the Standard Model (black star). The solid blue line indicates the anticipated constraint from the planned $Q_{W}^{p}$ measurement, assuming the SM value.

Beyond tree level, the weak charge of the proton can be written as $Q_{W}^{p}=\rho_{P V}\left(1-4 \kappa_{P V} \sin ^{2} \theta_{W}\right)+$ $\lambda_{p}=-2\left(2 C_{1 u}+C_{1 d}\right)$, where $\rho_{P V}, \kappa_{P V}$, and $\lambda_{p}$ are parameters that contain both radiative corrections within the Standard Model, as well as contributions from possible new physics [ए2]. The Standard Model radiative corrections for $Q_{W}^{p}$ include terms from $\gamma Z, Z Z, W W$ box and other loop diagrams. Additional radiative corrections associated with fermion and massive vector boson loops collectively give rise to the scale dependence (running) of the weak mixing angle, as seen in Fig $\mathbb{m}$. The quark weak charges relevant for the $Q_{W e a k}^{P}$ experiment are $C_{1 u}=\frac{1}{2} \rho_{e q}^{\prime}\left(1-\frac{8}{3} \kappa_{e q}^{\prime} \sin ^{2} \theta_{W}\right)+\lambda_{1 u}$

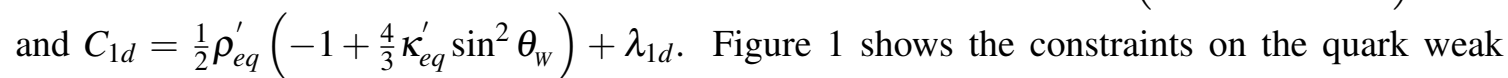
charges, placed by the indicated completed measurements (From $[Q]$ ). Combining the projected $Q_{W}^{p}$ measurement (in blue) with the previous experimental results will therefore lead to a significant improvement in the allowed range of values for $C_{1 u}$ and $C_{1 d}$. Assuming the Standard Model holds, the resulting new limits will significantly constrain new physics to be above a mass scale of $\approx 2 \mathrm{TeV}$, for new weakly coupled physics.

\section{Experimental Overview}

The experimental layout is shown in Fig. ㅁ. The experiment itself consists of well established technology. However, due to the high precision goal, several techniques were pushed to levels beyond where any other experiment has performed to date, both in precision and capacity. Examples of this include the target, which is the world's highest power liquid hydrogen $\left(\mathrm{LH}_{2}\right)$ target used in an electron scattering experiment [ए్] , a very fast electron beam helicity reversal rate of $960 \mathrm{~Hz}$ (see below), and a very high electron polarization of $89 \%$. See [四] and references within for a full description of the experiment. 

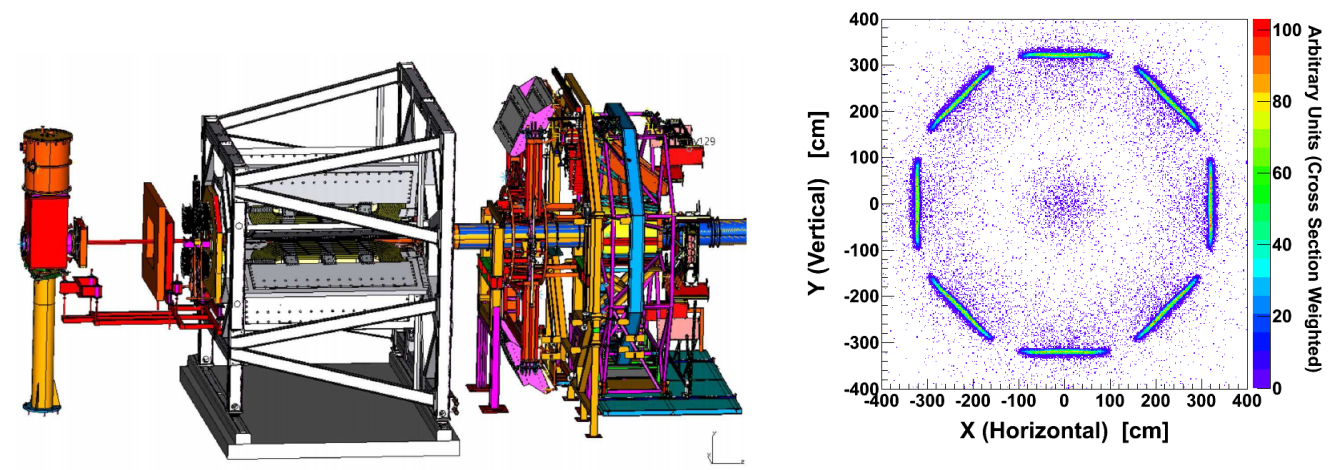

Figure 2: Left: Engineering drawing of the experimental layout. The beam runs from left to right in the figure. Right: Simulated event pattern in the focal plane for all eight octants. Only elastic events are shown.

The measured asymmetry for a given detector PMT can be written in the following way:

$$
\begin{gathered}
A_{m s r}=\frac{Y^{+}-Y^{-}}{Y^{+}+Y^{-}}+A_{\text {beam }}+A_{\varepsilon}=P\left(f_{p} A_{P V}(\vec{e}, p)+\sum_{b} f_{b} A_{b}\right) \\
Y^{ \pm}=Y^{o}\left(1 \pm A_{\text {raw }}\right) \pm \varepsilon .
\end{gathered}
$$

Here, $Y^{ \pm}$is the total signal yield seen in a given PMT for a right-handed (+) or left-handed () electron beam helicity state.The factors $f_{p}=\frac{\left\langle Y_{p}\right\rangle}{\langle Y\rangle}$ and $f_{b}=\frac{\left\langle Y_{b}\right\rangle}{\langle Y\rangle}$ are the fractional physics of interest and background contributions, respectively, to the total yield for the PMT. $P$ is the beam polarization, the $A_{b}$ are various background asymmetries, and $A_{\text {beam }}=A_{\text {beam }}\left(E, x, y, x^{\prime}, y^{\prime}\right)$ is the false asymmetry due to helicity correlated beam changes. The latter includes yield changes due to beam position $(x, y)$, beam angle $\left(x^{\prime}, y^{\prime}\right)$, and beam energy $(E)$ on target and includes an asymmetry from residual transverse polarisation. The total yield is normalized to the beam current. The $A_{\varepsilon}$ and $\varepsilon$ terms are electronic contributions from a small (but possible) helicity signal leakage into the data acquisition (DAQ) electronics or the detector pedestal, as well as from small PMT nonlinearities. The experiment was then designed to make high precision measurements of the beam polarization, the momentum transfer, and the signal yield. The experiment was also designed to suppress backgrounds and helicity correlated electronic and beam effects as much as possible, and experimental components were included in the design, to allow ancillary measurements of both background asymmetries and yields, as well as helicity correlated beam parameter changes.

The experiment made use of two polarimeters, a Compton polarimeter, used for continuous polarization measurements at full current, and a Møller polarimeter used for short invasive polarimetry two or three times a week at lower currents. Measurement of beam parameters was facilitated by a series of beam current monitors, beam position monitors, luminosity monitors, and beam halo monitors. From left to right in Fig. \, a $180 \mu \mathrm{A} 85 \%$ longitudinally polarized beam of $1.165 \mathrm{GeV}$ electrons is incident on a $\mathrm{LH}_{2}$ target with $2.5 \mathrm{~kW}$ of cooling power. The scattered electrons are collimated to define an average scattering angle of $\theta=8 \pm 2^{\circ}$ and an azimuthal acceptance of $53 \%$ of $2 \pi$. The collimators were carefully designed and constructed to produce a well known $Q^{2}$ range and suppress backgrounds. An eight sector toroidal magnet focuses the scattered 
electrons onto a set of eight, two meter long quartz Čerenkov bars. The scattered beam profile is shown on the right-hand side in Fig. $\square$.

The octagonal symmetry arrangement of the detectors allows for various choices of detector combinations that suppress helicity correlated beam motion effects when extracting the asymmetry from the signal. Four sets of wire chambers are used (two horizontal wire chambers (HDCs) before the magnet, at the collimators, and two vertical wire chambers (VDCs) in front of the detectors) to allow for calibration mode (at nA level beam currents) $Q^{2}$ determination and background measurements. For the $Q^{2}$ determination, the chambers provide two independent measurements of the electron tracks (before and after the spectrometer), which are connected in the analysis, using the accurately mapped spectrometer field. The HDCs are also used to determine the scattering vertex in the target.

At full current, the event rate per main detector is about $900 \mathrm{MHz}$ and they are therefore operated in current mode. The size of the main detectors ( 2 meters long, $18 \mathrm{~cm}$ wide and $1.25 \mathrm{~cm}$ thick), their position $(\approx 3.8$ meter radial distance from the forward beam line and $\approx 5.6$ meters from the center of the magnet), and their angle with respect to the vertical $\left(0^{\circ}\right)$ were carefully chosen to maximize the elastic electron rate, maximize light output, and minimize backgrounds. The Čerenkov light is detected by two photomultiplier tubes (PMT) for each quartz bar; one on each side. A $2 \mathrm{~cm}$ thick lead pre-radiator is mounted in front of each quartz bar, to increase the light yield from scattered electrons, using showering, and to decrease the signal from photon backgrounds. The experimental asymmetry must be corrected for backgrounds from electrons scattering off of the aluminum target windows, for backgrounds from inelastically scattered electrons as well as for soft photon and neutron background. Careful measurements of these backgrounds were done either through ancillary data runs, at various spectrometer field settings and beam energies, or insitu, using background detectors placed in various locations around the main detectors.

\section{Analysis and Results}

The data analysis for the asymmetry, polarization, and $Q^{2}$ measurements for the full data set is ongoing. The commissioning data $(4 \%)$ of the experiment has been published elsewhere [四]. The primary technology used in the clean extraction of the asymmetry in Eqn. $\square$. reversal, which greatly reduces effects due to possible signal changes, such as slow gain drifts, target density fluctuations, and beam drifts. The TJNAF laser source can accommodate high helicity reversal rates with high polarization, using a Pockels cell. $Q_{W e a k}^{P}$ routinely ran at a $960 \mathrm{~Hz}$ helicity reversal rate, coupled with a reversal pattern of $(+--+)$ or $(-++-)$ for consecutive helicity windows and with the initial window polarity changed pseudo-randomly. These patterns remove linear drifts in the signal and the fast reversal makes the approximation of relatively slow random fluctuations as linear drifts valid.

All remaining asymmetries are true systematic effects, such as those associated with helicity correlated beam changes and background physics. Sensitivities of the detectors to helicity correlated beam properties are measured in dedicated beam modulation runs, as well as from natural beam motion, during routine data production. These sensitivities are then used in regression, when the actual physics asymmetry of interest is extracted from the data. Additional helicity reversal techniques, so called slow reversal, are used to cancel systematic effects introduced in the source 
(e.g. at the photo cathode or in the Pockels cell). These include insertion of one or two half-wave plates after the Pockels cell, approximately every four hours, and a Wien electron spin rotation in the injector beam every few weeks. When a half-wave plate is inserted or a Wien flip is performed, the polarity (sign) of the asymmetry changes and the consistency with which this happens, without any changes in the size of the asymmetry, is an important measure of the systematic integrity of the experimental data.

\section{Conclusion}

The $Q_{\text {Weak }}^{p}$ collaboration has successfully completed a two year long measurement program with very high statistics and excellent control of systematic effects. This constitutes the first measurement of the proton weak charge and a search for new physics beyond the Standard Model up to mass scales of $\simeq 6 \mathrm{TeV}$ (model dependent). The collaboration is currently working on the completion of the data analysis.

\section{References}

[1] D. Androic et. al., Phys. Rev. Lett., 111:141803 (2013)

[2] M. J. Musolf, T.W. Donnelly, J. Dubach, S. J. Pollock, S. Kowalski, and E. J. Beise, Phys. Rep. 239, 1 (1994).

[3] W. J. Marciano and A. Sirlin, Phys. Rev. D 27, 552 (1983); Phys. Rev. D 29, 75 (1984); Phys. Rev. D 31, 213(E) (1985).

[4] J. Erler, A. Kurylov, and M. J. Ramsey-Musolf, Phys. Rev. D 68:016006 (2003)

[5] J. Erler and M. J. Ramsey-Musolf, Phys. Rev. D, 72:073003 (2005)

[6] J. Erler and M. J. Ramsey-Musolf. Prog. Part. Nucl. Phys., 54 351-442, (2005).

[7] M. J. Musolf et al., Phys. Rep. 239, 1 (1994).

[8] J. Roche, R. D. Carlini, R.D. Young, and A.W. Thomas, Phys. Rev. Lett., 97:102002, (2006).

[9] R. D. Carlini, A.W. Thomas, R.D. Young, and J. Roche, Phys. Rev. Lett., 99:122003, (2007).

[10] P. G. Blunden, W. Melnitchouk, and A.W. Thomas, Phys. Rev. Lett. 107:081801, (2011).

[11] A. Sibirtsev, P. G. Blunden, W. Melnitchouk, and A.W. Thomas Phys. Rev. D 82:013011, (2010).

[12] A. Kurylov, M.J. Musolf, S. Su Phys. Rev. D 68, 035008, (2003).

[13] M. Gorchtein, C. J. Horowitz, and Michael J. Ramsey-Musolf Phys. Rev. C 84:015502, (2011).

[14] B. C. Rislow and C. E. Carlson Phys. Rev. D 83:113007, (2011).

[15] S. C. Bennett and C. E. Wieman. Phys. Rev. Lett., 82:2484-2487, (1999).

[16] P. L. Anthony et al. Phys. Rev. Lett., 95:081601, (2005).

[17] G. P. Zeller et al. Phys. Rev. Lett., 88:091802, (2002).

[18] W. M. Yao et al. J. Phys., G33:1-1232, (2006).

[19] G. Smith, In the proceedings of the PAVI11 conference, From Parity Violation to Hadronic Structure and more, IL NUOVO CIMENTO, DOI: 10.1393/ncc/i2012-11297-2, (2012) 